\title{
DEMONSTRATION OF HOMOLOGOUS RECOMBINATION EVENTS IN THE EVOLUTION OF BOVINE VIRAL DIARRHOEA VIRUS BY IN SILICO INVESTIGATIONS
}

\author{
Csaba KÖVÁGÓ ${ }^{1 *}$, Ákos HoRNYÁK ${ }^{2}$, Violetta KÉKESI ${ }^{3}$ and Miklós RUSVAI ${ }^{4}$ \\ ${ }^{1}$ Department of Pharmacology and Toxicology and ${ }^{4}$ Department of Pathology, University \\ of Veterinary Medicine, István u. 2, H-1078 Budapest, Hungary; ${ }^{2}$ National Food Chain \\ Safety Office, Veterinary Diagnostic Directorate, Budapest, Hungary; ${ }^{3} \mathrm{Heart}$ and \\ Vascular Centre, Semmelweis University, Budapest, Hungary
}

(Received 2 February 2016; accepted 4 May 2016)

\begin{abstract}
Complete genome sequences of bovine viral diarrhoea virus types 1 and 2 (BVDV-1 and 2) deposited in the GenBank were submitted to bioinformatic analysis using a recombination-detecting software. The results indicate that recombination events are not rare in the case of BVDV, which frequently causes immunotolerance and, consequently, persistent infection in calves. The lack of specific immunity provides an ideal possibility for multiple infections by antigenically related but genetically different BVDV strains, and hence recombinations may occur. Among the $62 \mathrm{BVDV}-1$ genomes five recombinants and their possible parent strains, while among the 50 BVDV-2 genomes one simple recombinant and its parent strains were identified, which were supported by extremely strong probability values ( $\mathrm{P}$ values varying between $1.26 \times 10^{-4}$ and $1.58 \times 10^{-310}$ ). Besides the newly identified recombinants, recombination events described previously were confirmed, but in some of these cases former information was completed with new data, or different parent(s) were suggested by the programme (RDP 4.46 BETA) used in this study.
\end{abstract}

Key words: Genomic recombination, bovine viral diarrhoea virus, BVDV, RNA

Bovine viral diarrhoea virus is a worldwide pathogen causing severe economic losses in the cattle industry. The clinical presentation can range from inapparent or subclinical infection through acute and severe enteric disease to the highly fatal mucosal disease complex characterised by profuse enteritis in association with typical mucosal lesions. In the epizootiology of the disease the most important role is played by cattle persistently infected with non-virulent (noncytopathic) BVDV, because these animals serve as natural reservoirs for the virus. Persistent infection develops when noncytopathic BVDV is transmitted transplacentally during the first 4 months of fetal development. The calf is born infected

*Corresponding author; E-mail: kovago.csaba@aotk.szie.hu; Phone: 0036 (1) 478-4167 
with the virus, remains infected for life, and usually is immunotolerant to the resident noncytopathic virus (Fulton et al., 2003). This pathomechanism offers a good chance for homologous recombination that is a basic mechanism for the genetic diversification of viruses. Recombination is the formation of chimaerical nucleic acid molecules from parental genomes (major and minor parents, referring to the relative size of the genomic part within the chimaerical nucleic acid), which commonly occurs with RNA viruses (Simon-Loriere and Holmes, 2011).

Since the persistently infected, immunotolerant animals are not able to mount an immune response, co-infection (which is the precondition of recombination) with other BVDV strains genetically different from the strain causing the persistent infection is possible and may even be rather frequent. Furthermore, the formerly widespread live vaccines (non-virulent strains) used for prevention could also cause parallel infections.

The size of the BVDV genome is approximately $12-13 \mathrm{~kb}$. Based on comparisons of nucleotide sequence in the viral RNA, there are two species (distinct genetic groups, previously also referred to as genotypes) of BVDV, which are termed BVDV type 1 and BVDV type 2, and both cytopathic and noncytopathic BVDV strains are represented in each species (Ridpath and Neill, 2000). BVDV-1 can be further divided into subgenotypes or genogroups based on differences in the nucleotide sequences within the $5^{\prime} \mathrm{NTR}, \mathrm{N}^{\mathrm{pro}}$ and E2 genes (Becher et al., 1999; Toplak et al., 2004; Vilcek et al., 2004).

The significance of heterologous, host genome-viral genome recombination (insertion of host genomic parts) is described and discussed in detail by others (Becher and Tautz, 2011) who have evaluated the importance of this event in the background of the pathogenicity and cytopathogenicity of BVD virus.

In this study, we report evidences of homologous recombinations by investigating full-genome BVDV-1 and -2 sequences deposited in the GenBank using phylogenetic reconstructions and statistically based multiple recombination detection methods.

\section{Materials and methods}

$B V D V$ genomes included in the study. A total of $112 \mathrm{BVDV}$ genomes (62 BVDV-1 and 50 BVDV-2) deposited in the GenBank as complete sequences or nearly complete sequences (the latter ones containing more than 11,500 nucleotides and missing only the 3'- and 5'-end non-translated regions) were used in this study. The sequences were deposited under the following accession numbers:

BVDV-1 strains: AB078950, AB078951, AB078952, AF041040, AF091605, AF220247, AF268278, AF526381, AJ133738, AJ133739, AJ585412, DQ088995, EF101530, HQ174292, HQ174293, HQ174294, HQ174295, HQ174296， JN380080，JN380083， JN380088， JN380089, 
JN400273, JN644055, JN704144, JQ418633, JQ418634, JQ799141, JX297512, JX297513, JX297514, JX297515, JX297516, JX297517, JX297518, JX297519, JX297520， JX297521， JX419397， JX419398， KC695810， KC695814, KC757383， KC853440， KC853441， KC963967， KF501393， KF772785, KF835697, KF835698, KF835699, KF896608, KJ541471, KJ620017, KJ689448, M31182, M96687, M96751, LC089876, U63479, U86599, U86600.

BVDV-2 strains: AB567658, AB894423, AB894424, AF145967, AY149215, AY149216, FJ527854, GQ888686, HG426479, HG426480, HG426481, HG426482, HG426483, HG426484, HG426485, HG426486, HG426487, HG426488, HG426489, HG426490, HG426491, HG426492, HG426493, HG426494, HG426495, HQ174297, HQ174298, HQ174299, HQ174300, HQ174301, HQ174302, HQ174303, HQ258810, JF714967, JN380081, JN380082, JN380084, JN380085, JN380086, JN380087, JN380090, JQ418635， KC963968， KF835700， KF835701， KF835702， KJ000672, LC006970, NC002032, U18059.

Genetic alignment and construction of phylogenetic trees. Before phylogenetic analysis, multiple alignment was performed using the ClustalW method. Phylogenetic trees were constructed using the maximum-likelihood method and evaluated using the interior branch test method with Mega 6.06 software (Tamura et al., 2013). For bootstrap evaluation 1000 repetitions were calculated, the resulting bootstrap values are indicated at the nodes, GenBank accession numbers are at the end of each branch (Fig. 1).

Recombination detection. For detecting possible recombinant sequences, the alignment was submitted to the RDP 4.46 BETA software, containing several detection algorithms (Martin et al., 2015). As for the detection methods, we utilised the following: RDP (Martin and Rybicki, 2000), GENECONV (Padidam et al., 1999), Bootscan (Martin et al., 2005), MaxChi (Smith, 1992), Chimaera (Posada, 2002), SiScan (Gibbs et al., 2000), 3Seq (Boni et al., 2007) and LARD (Holmes et al., 1999). The size of the shifting window was 200 bases.

Recombination 'hot-spot' detection. For detecting possible regions where recombination events may occur at a high rate, we used the inbuilt breakpoint Pdistribution plot of RDP 4.46 BETA.

\section{Results}

The phylogenetic tree constructed using the full genome sequences of the BVDV strains deposited in GenBank is shown in Fig. 1. To avoid crowding, the tree does not show the accession numbers of the genomes that were homologous or nearly homologous with another strain present on the tree, showing higher than 98\% identity. Probable recombination events are indicated with arrows (Fig. 1). 


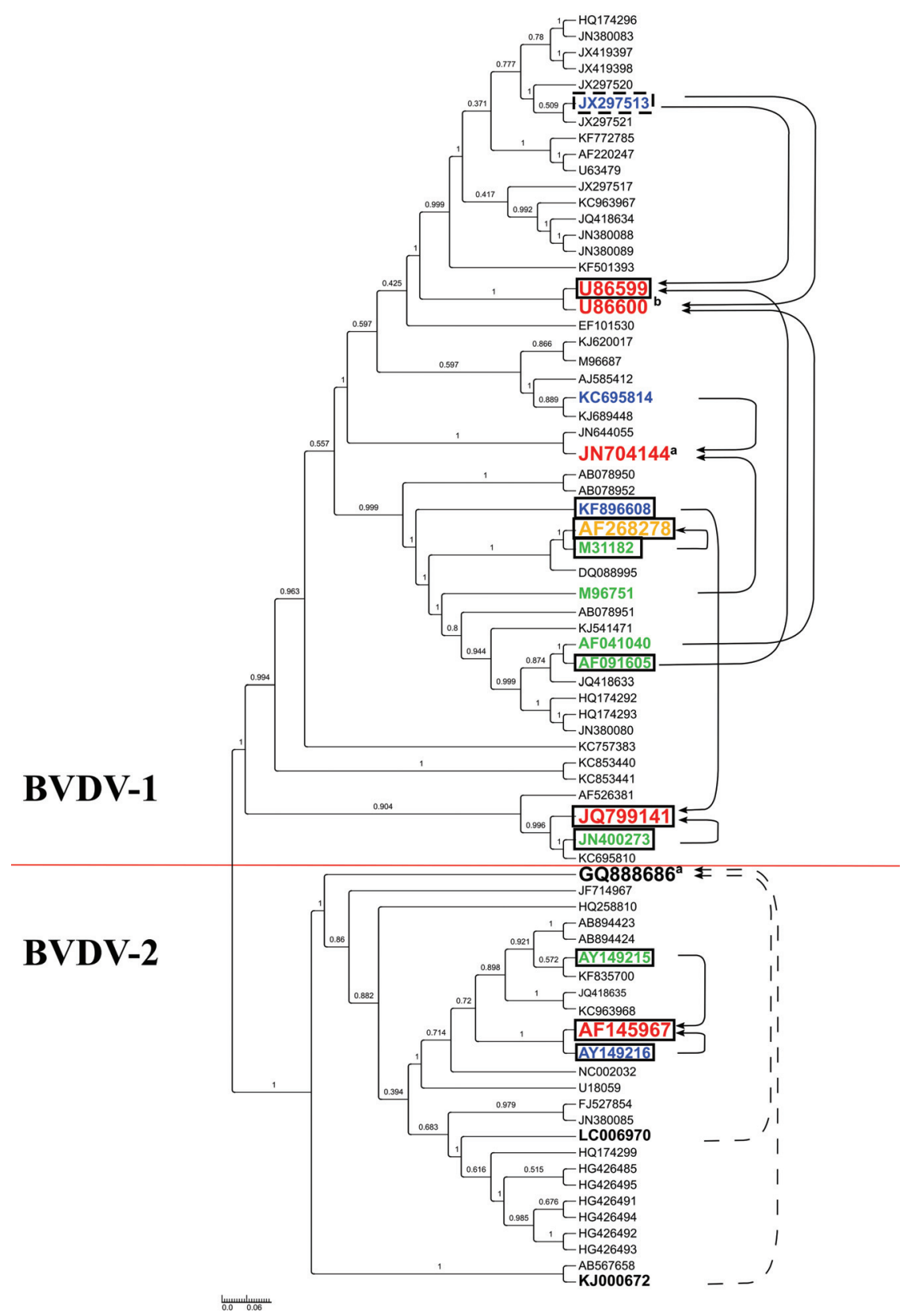

Fig. 1. Phylogenetic tree constructed using sequences selected from the 112 BVDV-1 and -2 full genome sequences deposited in the GenBank. Only one of the genomes was used if higher than $98 \%$ identity was indicated between two sequences, therefore only 72 representative genomes are on the tree. Red: recombinants, Blue: major parent, Green: minor parent. Orange: artificial chimaera strain, used as internal control. Recombinants and parents identified by our survey are framed. ${ }^{a}$ (Weber et al., 2015), ${ }^{\mathrm{b}}$ (Jones and Weber, 2004) 
The RDP graphs demonstrating the approximate size and localisation of the recombinations found by the RDP 4.46 BETA software are shown in Fig. 2A-E, while the exact localisation of the changed genomic parts is listed in Table 1. Detailed data on the recombinants, major and minor parents are shown in Table 2.

Among the 62 BVDV-1 genomes five recombinants were detected by the programme, one of which was double recombination (JN704144). One of the further three recombinations was an artificial chimaera (AF268278), while from the three remaining recombinations two (U86599 and U86600) are cp and ncp variants of genetically closely related strains. The last BVDV-1 recombinant (JQ799141) was isolated from a yak, and its major parent strain originated from cattle while the minor was detected in a pig.

From the 50 BVDV-2 strains only one recombinant (AF145967) was detected by our method. Another recombination event described previously by other authors (GQ888686, Weber et al., 2015) was not confirmed by our results.

Recombination breakpoint p-distribution plot analysis of the genomes detected four hot-spots (A: $0.5 \mathrm{~kb}, \mathrm{~B}: 1 \mathrm{~kb}, \mathrm{C}: 7.5 \mathrm{~kb}, \mathrm{D}: 12 \mathrm{~kb}$ ) on the BVDV-1 genome, which correlate with the detected recombinations rather well (Fig. 3A). Recombination can be identified more exactly when only the recombinant and the two parents are compared, but when all 62 BVDV-1 genomes were fed into the programme used for hot-spot analysis (RDP 4.46 BETA), the minor differences in the localisation of the breakpoints and the different length of the genomes resulted in relatively longer sequences; they may vary with a few hundred bases in the case of the different recombinants. Two similar hot spots are also detected in the BVDV-2 genome, but their location is slightly different compared to that of BVDV-1, they are around $0.15 \mathrm{~kb}$ and $11.7 \mathrm{~kb}$ (Fig. 3B).

\section{Discussion}

It was surprising that the ratio of recombinants in the case of BVDV was relatively high: five from the $62 \mathrm{BVDV}-1$ (8\%) and one from the $50 \mathrm{BVDV}-2$ $(2 \%)$ genomes. All these recombinations were supported with extremely strong $\mathrm{P}$ values (varying between $1.58 \times 10^{-310}$ to $1.26 \times 10^{-4}$ ), which indicates an almost absolutely proven recombination event in the case of these viruses. From the six recombinants detected by us, two had already been described by other authors, but additional information on some of them was gained by our investigations. We decided to use only full or nearly full sequences, because the shorter sequences are used for recombination search, the more events are found, but the shorter sequences increase the probability of false positive findings, since similarities on short regions may be the consequences of mutations as well. 

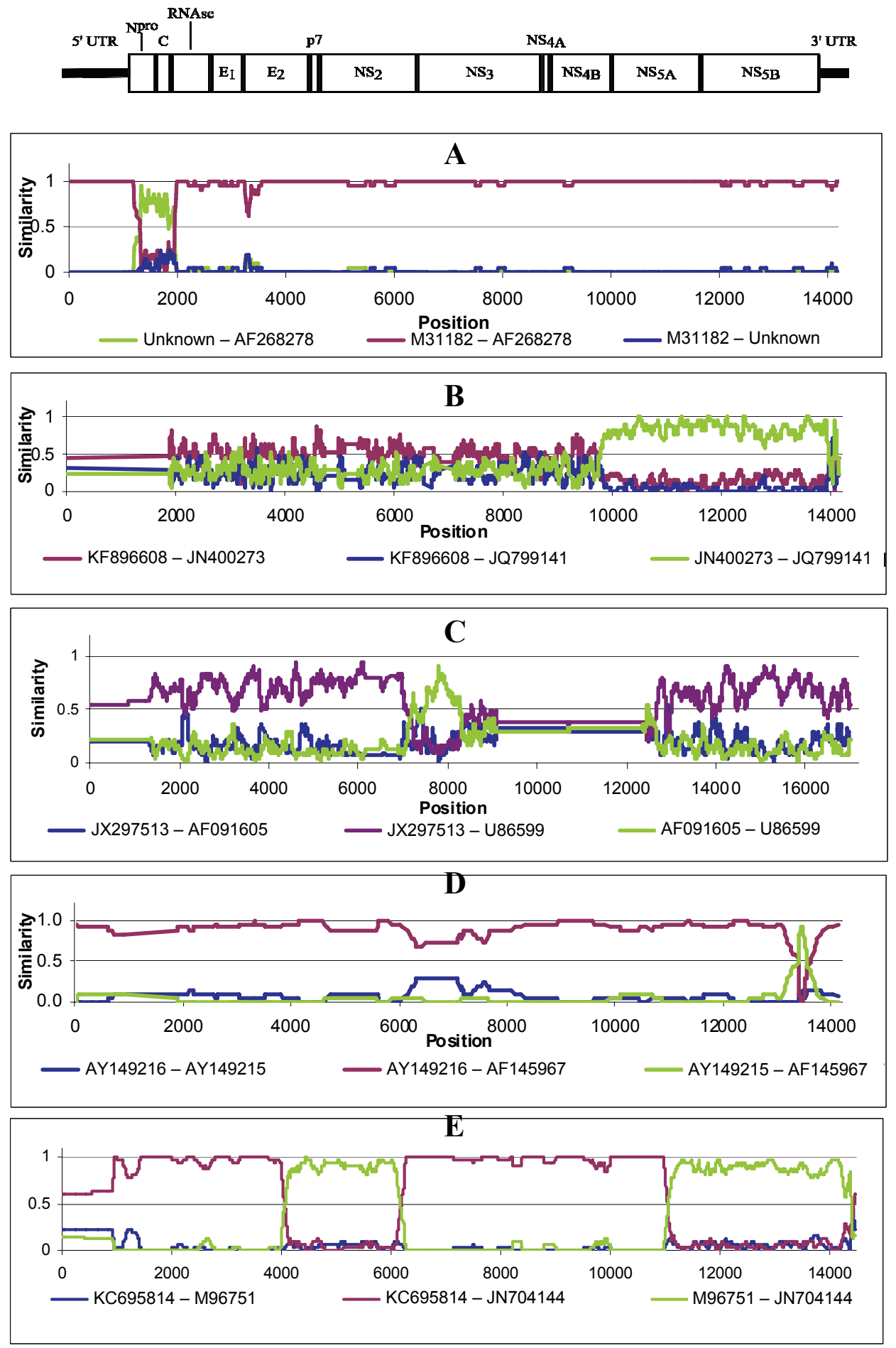

Acta Veterinaria Hungarica 64, 2016 
Although the full genome length of BVDV may be only slightly longer than $13 \mathrm{~kb}$, the scale of the graphs (Fig. 2) is longer than that (14.2 kb) due to the shifts and gaps in the alignments, and due to aligning BVDV-1 and -2 genomes in the same investigation. (This latter could not be avoided, since we wished to investigate the possibility of cross-type recombinations, too.) This 'wobbling' of the actual genomes in the alignment results in a theoretical genome size which is longer than any of the sequences, therefore the graphs prove and illustrate only the event of the recombination, but the exact position of the changed part cannot be read accurately from the graphs, only approximately. On the other hand, the programme localises the start- and endpoints exactly; those are shown in Table 1.

The two species of BVDV are clearly separated on the phylogenetic tree. One interspecies recombination between BVDV-1 and -2 was detected (Fig. 2D), which supports previous observations regarding the possibility of recombination between the two BVDV species, though the recombination event described earlier (Ridpath and Bolin, 1995) was not detected when complete genomes were aligned. The low probability of interspecies recombinations supports the hypothesis that immunotolerance (which is type specific) increases the probability of co-infections with BVDV strains of the same species, therefore helps recombination within the same species. On the other hand, there are recombinations among the different subtypes of BVDV-1 (BVDV-1a-g) identified by previous authors (Becher et al., 1999; Vilcek et al., 2004), though the location of subgroups on the branches of the tree is not always supported when full genomes are used, not only the 5' end region (5'-UTR, $\mathrm{N}_{\text {pro }}$ ) of the BVDV genome.

In our study, the programme selected those deposited sequences as parent sequences, which were nearest to the probable parents. This does not mean that the recombinants originated really from these parents, since the real donors were most probably not sequenced and deposited in the GenBank. Therefore, the respective genomic regions of the parents and the derived recombinants are not identical nucleotide by nucleotide, only very closely related on the exchanged genomic regions.

A good example of that is the AF268278 (BVDV-1a) recombinant strain. Only one of its parents (M31182) could be detected, because the recombinant virus is an artificial chimaera BVDV-1 strain, produced by replacing the BVDV $\mathrm{N}_{\text {pro }}$ gene with a human hepatitis $\mathrm{C}$ genomic segment (Lai et al., 2000). The re-

\footnotetext{
Fig. 2. RDP graphs of the full genome alignment (major parent and recombinant identity percentage: purple, minor parent and recombinant identity percentage: green, minor and major parent identity percentage: blue). A: recombinant AF268278. The major parent was identified as the NADL strain (M31182). The minor parent is unknown. B: recombinant JQ799141 and minor parent JN400273 and major parent KF896608. C: recombinant U86599 and minor parent AF091605 and major parent JX297513. D: AF145967 and minor parent AY149216 and major parent AY149215. E: double recombinant JN704144 and minor parent M96751 and major parent KC695814, respectively
} 
combination is presented in Fig. 2. This partial replacement caused an 'unknown' result of the search in case of the minor parent, since hepatitis $\mathrm{C}$ genomes were not included in the alignment as possible parents. At the same time, this finding proves the robustness of the applied test methods.

The programmes confirmed the results of Weber et al. (2015) by revealing a possible double cross-recombinant (JN704144), which strain was demonstrated in China from a field case (Fig. 2A) (Weber et al., 2015). The major parent is the Av69 Vedevac strain also sequenced in China, the minor parent is a field strain (SD1). Besides being an example of a presumably rare double recombination event, this strain clearly demonstrates the risks of applying traditional attenuated live vaccines to reduce economical losses. Also, this recombination is unique in the extremely high probability of recombination, because using the different detection methods the $\mathrm{P}$ values are very strong, they vary between $\mathrm{P}<7.23 \times 10^{-43}$ and $1.28 \times 10^{-230}$ (Table 1 ).

Another recombinant, JQ799141, is also a Chinese strain as well as its parent strains JN400273 and KF896608 (Fig. 2). It is interesting that the recombinant itself was isolated from a yak, while one of the parents, JN400273, was isolated from swine (Deng et al., 2014). The strain must also circulate in cattle, otherwise the recombination could not occur; but the species variety among the two parents and the recombinant (cattle, swine, yak) is remarkable from the epizootiological point of view. Previous authors publishing results on recombination events did not report on recombinations between different viral species of different hosts (border disease virus of sheep, bovine viral diarrhoea virus of cattle and classical swine fever virus of pigs) within the Pestivirus genus. Although in our investigations we also included $12 \mathrm{BDV}$ (border disease virus) and $171 \mathrm{CSF}$ (classical swine fever) genomes, neither could we detect interspecies recombinations with viruses of other host species, and therefore the accession numbers of the used CSF and BDV strains were not listed in the Materials and methods. Nonetheless, the origin and existence of JQ799141 suggest that interspecies recombinations theoretically may appear later.

U86599 is also interesting for more than one reason. A very similar strain (U86600) was previously identified (Jones and Weber, 2004) as a recombinant using SimPlot analysis. The major parent was the same in our investigations as well (JX297513), but the minor parent was different (AF091605 instead of AF041040), though very closely related to the one identified as minor parent in the previous study. Besides finding another minor parent, the more sophisticated programmes used in our study found a longer recombination region and stronger $\mathrm{P}$ values in U86599, which proves that this strain was a recombinant, and U86600 is a derivate of U86599 different in less than $1 \%$ of the nucleotides, which may be the consequence of point mutations. Another interesting feature of this strain is that it is the least supported recombination in our study (Fig. 2C); the $\mathrm{P}$ values were not as strong as in the case of the other chimaeras, what is more, 


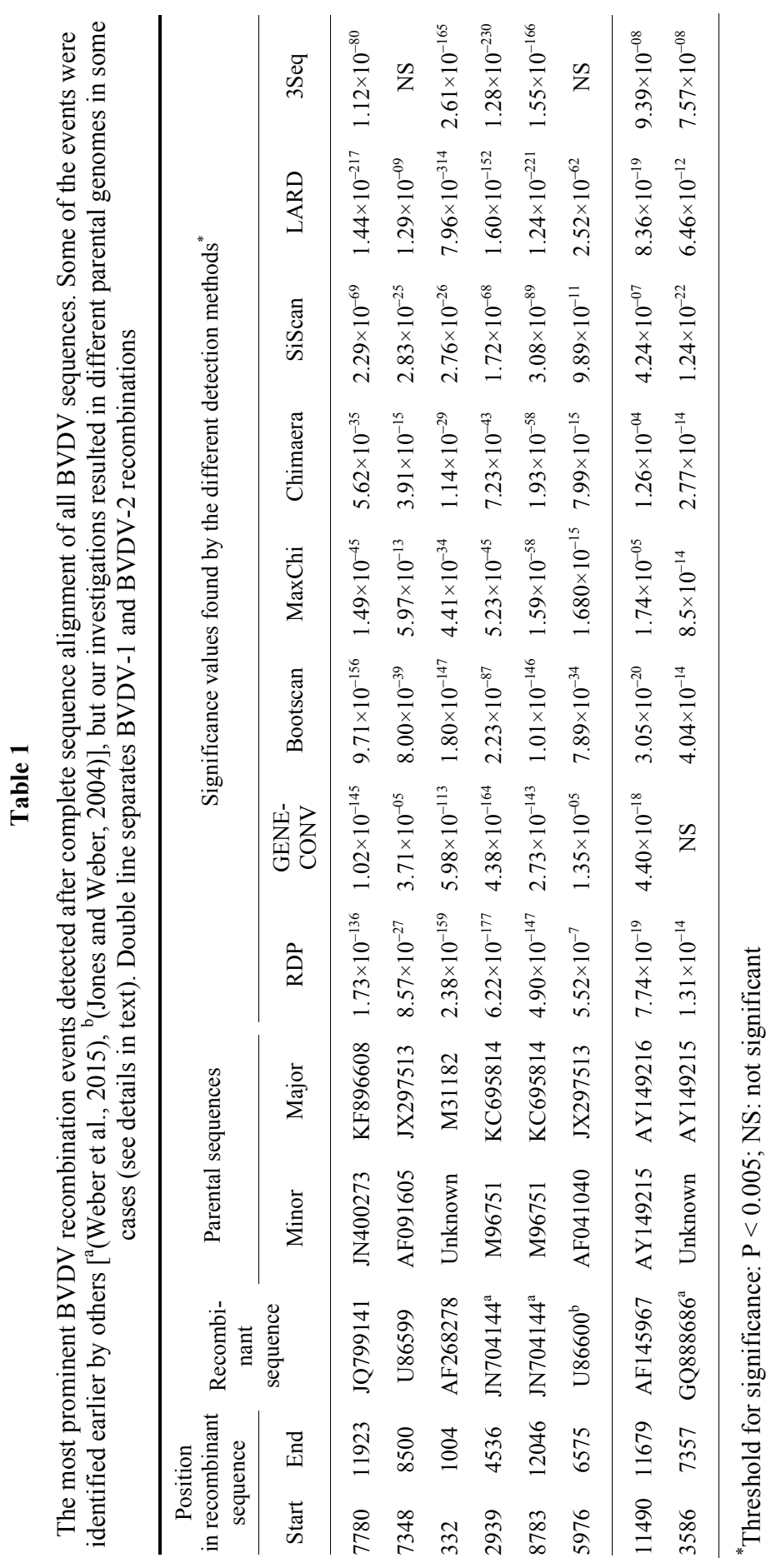




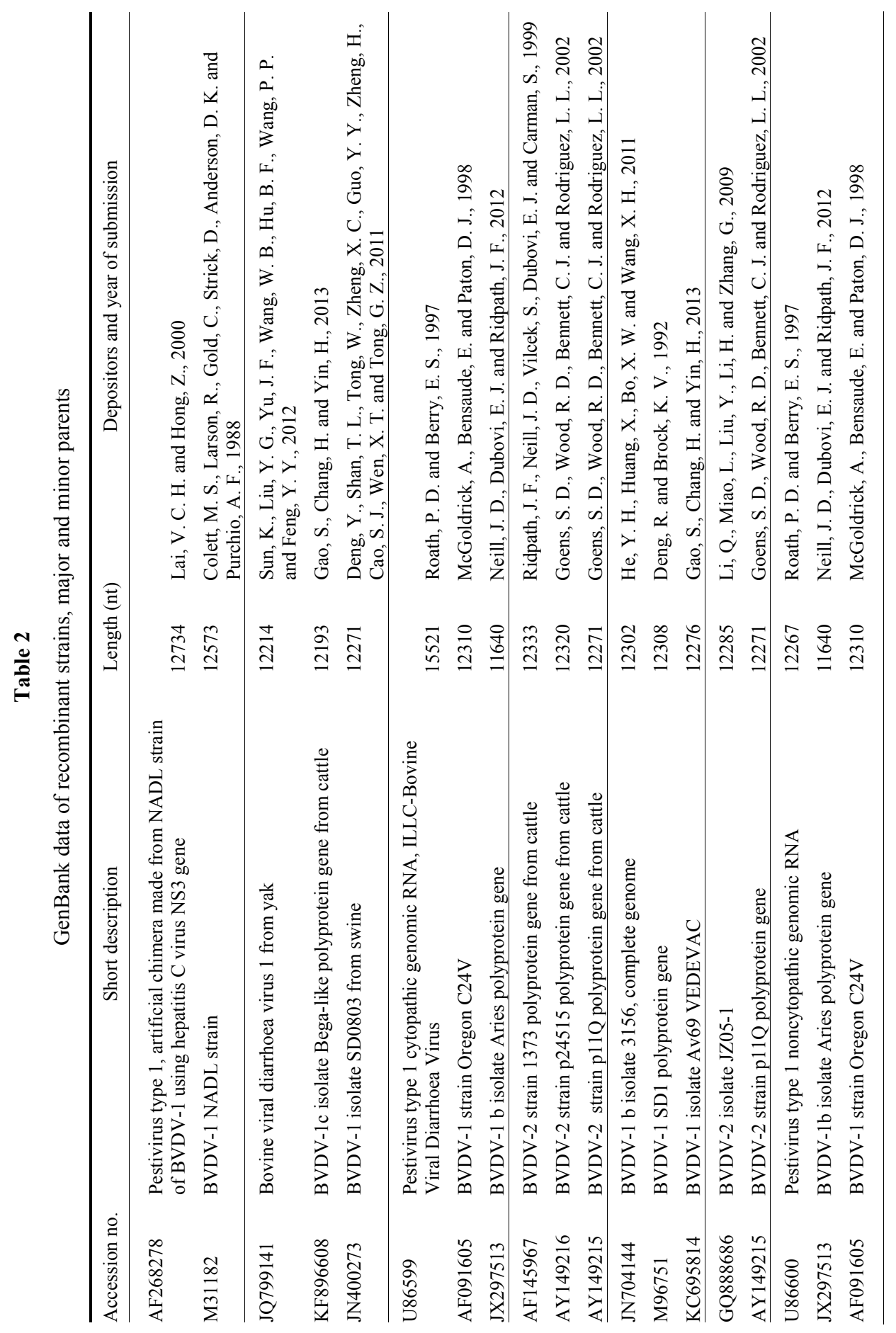



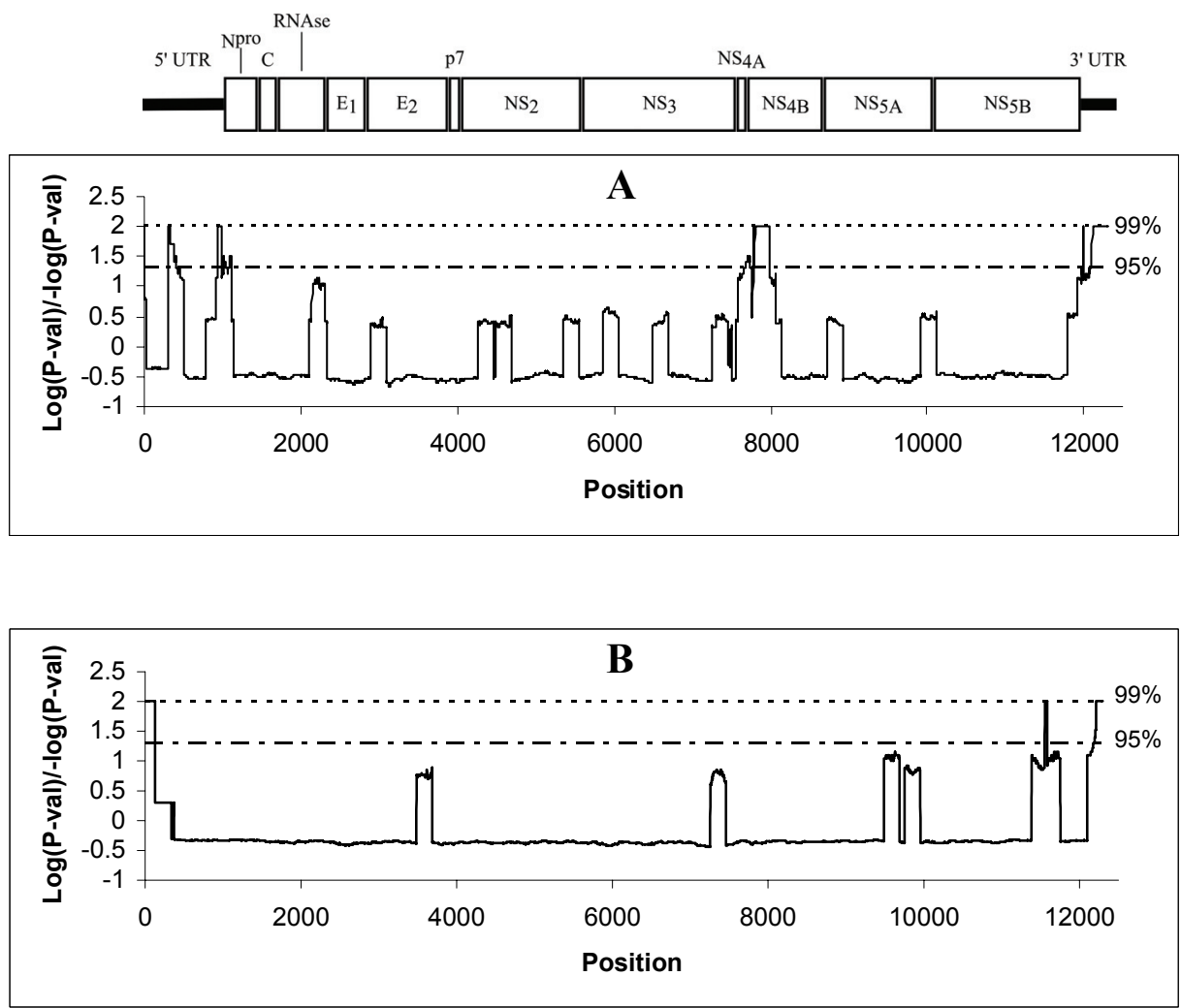

Fig. 3. These graphs demonstrate the results of the recombination 'hot-spot' analyses. Breakpoints are referred to the general BVDV genome, simplified genome structure is shown above the graph, dotted and dash-dotted lines in the graphs are representing confidence levels. A: Breakpoint pdistribution graph resulted from the BVDV-1 genomes alignment. B: Breakpoint p-distribution graph resulted from the BVDV-2 genomes alignment

one of the tests did not reveal recombination in this case, the $\mathrm{P}$ value was below the threshold for the 3 Seq method. We decided to introduce this event to prove that the results of the tests must be treated with consideration; comparison of the results of the different methods and visual re-evaluation of the graphs are always necessary, as it was suggested previously (Leal et al., 2012). The major parent (Neill et al., 2015) was demonstrated in the USA, the closest minor parent in the UK. Since close relatives of the real parents were not sequenced in this case, the $\mathrm{P}$ value is relatively low. It is unique in the length of the total genome $(15,521$ $\mathrm{kb}$ ) which is the consequence of three repetitions in its genome within the NS2-3 region, but these are most probably consequences of duplication of a $3263 \mathrm{nt}$ long sequence of a partially overlapping part of three genes (partial NS2, NS2-3, NS3) within the genome of the strain itself (Fig. 4), since the identity of the repetitive sequences is $98-100 \%$. This strain also draws the users' attention to the 
risk of false positive results since this strain was detected as a recombinant, though the repetitive regions are most probably of self origin. As the programme does not compare the genomes to themselves, repetitions will be detected as recombinations with the closest relative of the investigated strain, since the duplicated genomic region shows the highest identity to the closest relative.

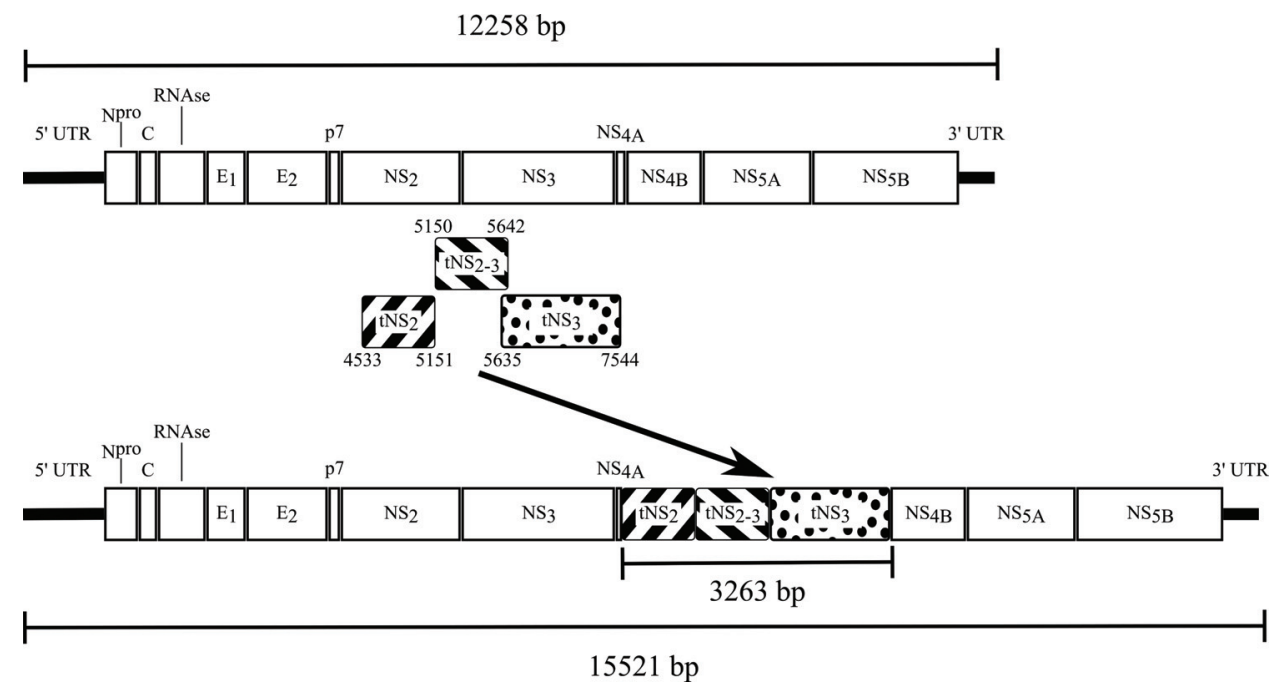

Fig. 4. Inserted repetitive sequences in the genome of strain U86599

A good example emphasising the need of re-evaluation of the results given by the software is a BVDV-2 recombination event described by Weber et al. (2015) who reported a putative recombinant (GQ888686), with relatively weak P values in certain tests (Table 1), and one of the applied methods did not even detect recombination at all. Using all 112 genomes in our study and a threshold limit of $\mathrm{P}=0.005\left(5 \times 10^{-3}\right)$ we did not identify this strain as a recombinant. When parameters were changed and merely 72 representative genomes were analysed (see the phylogenetic tree in Fig. 1) the programme found this recombinant, but the supporting $\mathrm{P}$ values were relatively weak (Table 1). We did not consider this strain as a recombinant.

It is interesting that relatively less recombinations were found in the case of BVDV-2, though the number of the deposited sequences was nearly as high as in the case of BVDV-1. Furthermore, most BVDV-2 strains were demonstrated and sequenced in North America (USA and Canada), which means that these virus variants coexisted in the same restricted geographical region, which increases the possibility of simultaneous infections. It may prove the relative stability of BVDV-2 compared to BVDV-1, which is also indicated by the lower genetic variance, and fewer putative subgroups in this viral species (Frey et al., 1996). 
Besides the recombinant described by Weber et al. (2015) mentioned above and not approved as a recombinant by us, the only recombinant detected in our study was AF145967, introduced in Fig. 2. The recombinant was described by Ridpath et al. (2006), but they detected a recombination different from ours. They identified not a recombination but an insertion within the NS3 gene of the genome, which is signed by a smaller drop of identity percentage in the graph in our investigation (Fig. 2D) and was not confirmed as a recombination event by our results. We have detected a much more prominent recombination closer to the 3' end within the NS5b region, and it is interesting that the minor parent from which this 195-base-long part is derived was identified by the programme as AY149215 BVDV-2. Searching further, after cutting out these 195 bases, the BLAST algorithm (Altschul et al., 1990) search identified the origin of this partial sequence as a BVDV-1 strain which was deposited with the accession number KF896612 by Gao et al. in the GenBank in 2013. It seems that the minor parent recognised by our programme was a recombinant itself with major parent AY149216 and minor parent KF896612. This minor parent was not identified originally by RDP because partial sequences were not among the investigated genomes. The length of the recombined genomic part is $195 \mathrm{nt}$, the identity to KF896612 partial sequence was $100 \%$. So considering this event, the existence of interspecies recombinants is also supported by the identification method used in our study, though only between the two BVDV species infecting the same host (cattle).

Our results prove the relatively high frequency of recombination in the evolution of BVDV. Although reassortment is known to occur frequently in case of viruses with segmented genomes (i.e. influenza viruses, bluetongue virus), this is not so in virus families with unsegmented genomes. In the case of BVDV, the special pathomechanism and the occurrence of persistently infected animals in the endemically infected countries may facilitate intergenomic recombinations. Also, the value of utilising multiple statistical methods in the identification of such events is further supported.

\section{References}

Altschul, S. F., Gish, W., Miller, W., Myers, E. W. and Lipman, D. J. (1990): Basic local alignment search tool. J. Mol. Biol. 215, 403-410.

Becher, P. and Tautz, N. (2011): RNA recombination in pestiviruses: Cellular RNA sequences in viral genomes highlight the role of host factors for viral persistence and lethal disease. RNA Biol. 8, 216-224.

Becher, P., Orlich, M., Konig, M. and Thiel, H. J. (1999): Nonhomologous RNA recombination in bovine viral diarrhea virus: Molecular characterization of a variety of subgenomic RNAs isolated during an outbreak of fatal mucosal disease. J. Virol. 73, 5646-5653.

Boni, M. F., Posada, D. and Feldman, M. W. (2007): An exact nonparametric method for inferring mosaic structure in sequence triplets. Genetics 176, 1035-1047.

Deng, Y., Shan, T. L., Tong, W., Zheng, X. C., Guo, Y. Y., Zheng, H., Cao, S. J., Wen, X. T. and Tong, G. Z. (2014): Genomic characterization of a bovine viral diarrhea virus 1 isolate from swine. Arch. Virol. 159, 2513-2517.

Frey, H. R., Flebbe, U. and Liess, B. (1996): Prevalence and clinical symptoms of persistent BVDvirus infection in cattle herds of Lower Saxony. Prakt. Tierarzt 77, 49-52. 
Fulton, R. W., Step, D. L., Ridpath, J. F., Saliki, J. T., Confer, A. W., Johnson, B. J., Briggs, R. E., Hawley, R. V., Burge, L. J. and Payton, M. E. (2003): Response of calves persistently infected with noncytopathic bovine viral diarrhea virus (BVDV) subtype $1 \mathrm{~b}$ after vaccination with heterologous BVDV strains in modified live virus vaccines and Mannheimia haemolytica bacterin-toxoid. Vaccine 21, 2980-2985.

Gibbs, M. J., Armstrong, J. S. and Gibbs, A. J. (2000): Sister-scanning: a Monte Carlo procedure for assessing signals in recombinant sequences. Bioinformatics 16, 573-582.

Holmes, E. C., Worobey, M. and Rambaut, A. (1999): Phylogenetic evidence for recombination in dengue virus. Mol. Biol. Evol. 16, 405-409.

Jones, L. R. and Weber, E. L. (2004): Homologous recombination in bovine pestiviruses: Phylogenetic and statistic evidence. Infect. Genet. Evol. 4, 335-343.

Lai, V. C. H., Zhong, W. D., Skelton, A., Ingravallo, P., Vassilev, V., Donis, R. O., Hong, Z. and Lau, J. Y. N. (2000): Generation and characterization of a hepatitis C virus NS3 proteasedependent bovine viral diarrhea virus. J. Virol. 74, 6339-6347.

Leal, E., Villanova, F. E., Lin, W. C., Hu, F., Liu, Q. F., Liu, Y. B. and Cui, S. J. (2012): Interclade recombination in porcine parvovirus strains (Retracted article. See vol. 94, pg. 464, 2013). J. Gen. Virol. 93, 2692-2704.

Martin, D. and Rybicki, E. (2000): RDP: detection of recombination amongst aligned sequences. Bioinformatics 16, 562-563.

Martin, D. P., Murrell, B., Golden, M., Khoosal, A. and Muhire, B. (2015): RDP4: Detection and analysis of recombination patterns in virus genomes. Virus. Evol. 1 (1): vev003. DOI: http://dx.doi.org/10.1093/ve/vev003.

Martin, D. P., Posada, D., Crandall, K. A. and Williamson, C. (2005): A modified bootscan algorithm for automated identification of recombinant sequences and recombination breakpoints. AIDS Res. Hum. Retrov. 21, 98-102.

Neill, J. D., Dubovi, E. J. and Ridpath, J. F. (2015): Identification of amino acid changes in the envelope glycoproteins of bovine viral diarrhea viruses isolated from alpaca that may be involved in host adaptation. Vet. Microbiol. 179, 299-303.

Padidam, M., Sawyer, S. and Fauquet, C. M. (1999): Possible emergence of new geminiviruses by frequent recombination. Virology 265, 218-225.

Posada, D. (2002): Evaluation of methods for detecting recombination from DNA sequences: Empirical data. Mol. Biol. Evol. 19, 708-717.

Ridpath, J. F. and Bolin, S. R. (1995): The genomic sequence of a virulent bovine viral diarrhea virus (BVDV) from the type-2 genotype - detection of a large genomic insertion in a noncytopathic BVDV. Virology 212, 39-46.

Ridpath, J. F. and Neill, J. D. (2000): Detection and characterization of genetic recombination in cytopathic type 2 bovine viral diarrhea viruses. J. Virol. 74, 8771-8774.

Ridpath, J. F., Neill, J. D., Vilcek, S., Dubovi, E. J. and Carman, S. (2006): Multiple outbreaks of severe acute BVDV in North America occurring between 1993 and 1995 linked to the same BVDV-2 strain. Vet. Microbiol. 114, 196-204.

Simon-Loriere, E. and Holmes, E. C. (2011): Why do RNA viruses recombine? Nat. Rev. Microbiol. 9, 617-626.

Smith, J. M. (1992): Analyzing the mosaic structure of genes. J. Mol. Evol. 34, 126-129.

Tamura, K., Stecher, G., Peterson, D., Filipski, A. and Kumar, S. (2013): MEGA6: Molecular Evolutionary Genetics Analysis Version 6.0. Mol. Biol. Evol. 30, 2725-2729.

Toplak, I., Sandvik, T., Barlic-Maganja, D., Grom, J. and Paton, D. J. (2004): Genetic typing of bovine viral diarrhoea virus: most Slovenian isolates are of genotypes 1d and 1f. Vet. Microbiol. 99, 175-185.

Vilcek, S., Durkovic, B., Kolesarova, M., Greiser-Wilke, I. and Paton, D. (2004): Genetic diversity of international bovine viral diarrhoea virus (BVDV) isolates: identification of a new BVDV-1 genetic group. Vet. Res. 35, 609-615.

Weber, M. N., Streck, A. F., Silveira, S., Mosena, A. C. S., da Silva, M. S. and Canal, C. W. (2015): Homologous recombination in pestiviruses: Identification of three putative novel events between different subtypes/genogroups. Infect. Genet. Evol. 30, 219-224. 